\title{
Double UAV cooperative localization and remote location error analysis
}

\author{
Hu tian xiang \\ ( College of Mechatronic Engineering, Nanjing Institute of Industry Technology, Nanjing 210023, \\ China )
}

key words : UAV ; Double station location ; Difference location ; Distance Measurement

\begin{abstract}
In this paper, through the placement phase positioning equipment short-range observation station, the measured data of the geometric relationship of low precision ranging applications into the rear observation station, the current post observation station and the distance between the rear observation station to meet data calculation need, can realize the rear observation station of the remote high precision ranging targets, so as to achieve double cooperative reconnaissance and mapping work.
\end{abstract}

\section{Introduction}

Airborne station passive location technology in measuring star positioning accuracy, has yet to be improved. Especially for the remote realization of the high precision positioning may have difficulties at present. Even if is the use of multi-aircraft cooperative location method has certain degree of technical difficulty. According to the theory of error analysis. Multi-station TDOA location system of baseline length between stations must be at least more than ten kilometers. In order to obtain better positioning accuracy, need tens of kilometers long. There are so many questions: (1)Location of high PRF signal ambiguity ; (2) Multi-station cannot at the same time on the side of narrow beam signal target.

The difference between the short baseline positioning is difficult to obtain good positioning accuracy. Despite the use of long baseline positioning can be achieved by multi-station distance and high precision positioning. There are many technical problems to be solved in the airborne system. A large distance apart two cooperative passive location method. Short range close to the enemy target in front of the station can be used for short baseline simple difference measurement technology for UAV. Rear observation station can be a fighter. The existing mathematical model has been proved. With the help of the long baseline bistatic. It can realize the rear observation station remote high precision of target.

\section{Phase difference location}

As shown in Figure 1, a multi-channel interferometer for passive detection of targets using a one-dimensional double array. For phase unwrapping, which each array is composed of multi-baseline array. Such as the radial distance corresponding to each. The measured displacement unit. Distance measurement based on phase shift is: 


$$
r_{i}=\lambda\left(n_{i}+\frac{\phi_{i}}{2 \pi}\right) \ldots(i=1,2)
$$

In the formula: $\lambda$ is wavelength; $n_{i}$ is the wavelength integer.

According to the relationship between the phase shift and the distance ( 1 ). In the single line array element two the radial distance between the path difference can be determined by measuring the difference.

$$
\Delta r=r_{1}-r_{2}=\lambda\left(\Delta n+\frac{\Delta \phi}{2 \pi}\right)
$$

In the formula: $\Delta n=n_{1}-n_{2}$ is the path difference contains wavelength integer; $\Delta \phi=\phi_{1}-\phi_{2}$ is phase difference between the two element array. By cosine theorem can be listed as follows two geometry equations:

$$
\begin{aligned}
& r_{1}^{2}=r_{2}^{2}+d_{1}^{2}-2 d_{1} r_{2} \cos (90+\theta)=r_{2}^{2}+d_{1}^{2}+2 d_{1} r_{2} \sin \theta \\
& r_{3}^{2}=r_{2}^{2}+d_{2}^{2}-2 d_{2} r_{2} \cos (90-\theta)=r_{2}^{2}+d_{2}^{2}-2 d_{2} r_{2} \sin \theta
\end{aligned}
$$

Type: $\theta$ in short baseline weekly direction as a benchmark, the target signal reaches outpost azimuth; $d$ is array distance.

Because of $X=r_{2} \sin \theta$, it can be adapted to the geometric auxiliary equation:

$$
\begin{aligned}
& r_{1}^{2}=r_{2}^{2}+d_{1}^{2}+2 d_{1} x \\
& r_{3}^{2}=r_{2}^{2}+d_{2}^{2}-2 d_{2} x
\end{aligned}
$$

Hypothesis : $r_{i}-r_{i+1}=\lambda\left(\Delta n_{i}+\frac{\Delta \phi_{i}}{2 \pi}\right)=\Delta r_{i}$

In Formula: $\Delta \phi_{i}=\phi_{i}-\phi_{i+1}$

The type (5) and (6) into geometric assisted (3) and (4), and the expression of simple, direct $\Delta r_{1}$ measurement is used to express. In mathematics after finishing there are two yuan for a system of linear equations:

$$
\begin{aligned}
& 2 d_{1} x-2 \Delta r_{1} r_{2}=-d_{1}^{2}+\Delta r_{1}^{2} \\
& 2 d_{2} x-2 \Delta r_{2} r_{2}=d_{2}^{2}-\Delta r_{2}^{2}
\end{aligned}
$$

Can be directly solved from:

$$
x=\frac{\left(d_{1}^{2}-\Delta r_{1}^{2}\right) \Delta r_{2}+\left(d_{2}^{2}-\Delta r_{2}^{2}\right) \Delta r_{1}}{2\left(\Delta r_{1} d_{2}-\Delta r_{2} d_{1}\right)}
$$




$$
r_{2}=\frac{\left(d_{1}^{2}-\Delta r_{1}^{2}\right) d_{2}+\left(d_{2}^{2}-\Delta r_{2}^{2}\right) d_{1}}{2\left(\Delta r_{1} d_{2}-\Delta r_{2} d_{1}\right)}
$$

By the target position parameter, the angle of arrival of the target can be obtained:

$$
\sin \theta=\frac{x}{r_{2}}=\frac{\left(d_{1}^{2}-\Delta r_{1}^{2}\right) \Delta r_{2}+\left(d_{2}^{2}-\Delta r_{2}^{2}\right) \Delta r_{1}}{\left(d_{1}^{2}-\Delta r_{1}^{2}\right) d_{2}+\left(d_{2}^{2}-\Delta r_{2}^{2}\right) d_{1}}
$$

Note that the symbol is in fact a phase measurement term: $\lambda\left(\Delta n_{i}+\frac{\Delta \phi_{i}}{2 \pi}\right)$ the angle of $\theta$ arrival is obtained by a phase difference measurement. When adjacent baseline is equal:

$$
\begin{gathered}
r_{2}=\frac{2 d^{2}-\Delta r_{1}^{2}-\Delta r_{2}^{2}}{2\left(\Delta r_{1}-\Delta r_{2}\right)} \\
\sin \theta=\frac{x}{r_{2}}=\frac{\left(d^{2}-\Delta r_{1}^{2}\right) \Delta r_{2}+\left(d^{2}-\Delta r_{2}^{2}\right) \Delta r_{1}}{d\left(2 d^{2}-\Delta r_{1}^{2}-\Delta r_{2}^{2}\right)}
\end{gathered}
$$

\section{remote ranging equation}

Design based on one-dimensional double difference forward near detection station station to the rear of the remote detection and geometry relation between two stations before and after as shown in Figure 1, the direct use of the cosine theorem can be obtained by remote observation station to the target distance.

$$
r_{4}^{2}=r_{2}^{2}+D^{2}-2 r_{2} D \sin (\theta-\alpha)
$$

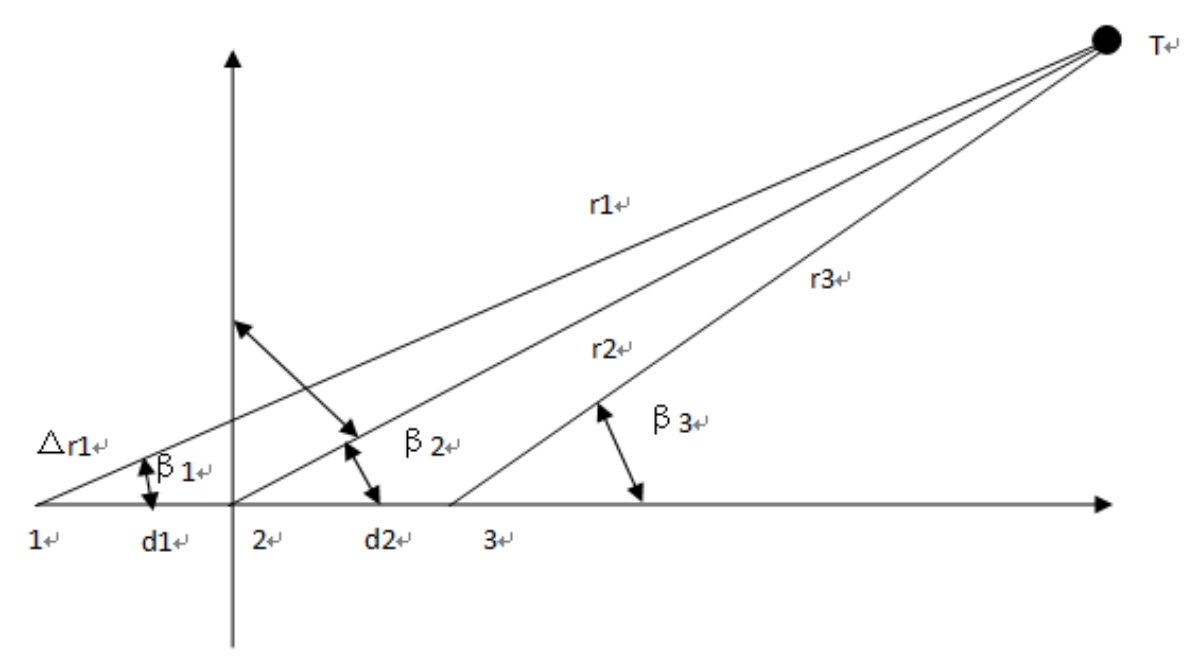

Figure 1 double difference location one-dimensional array

In the formula: $D$ it is the distance between the front and rear two observation stations, and the angle $\alpha$ between the baseline and the baseline direction of the two observation stations in the former observation station.

The type (12) (14) can be calculated by the target type and between remote stations in the radial distance:

$$
r_{4}=\sqrt{\left[\frac{2 d^{2}-\Delta r_{1}^{2}-\Delta r_{2}^{2}}{2\left(\Delta r_{1}-\Delta r_{2}\right)}\right]^{2}+D^{2}-\left[\frac{2 d^{2}-\Delta r_{1}^{2}-\Delta r_{2}^{2}}{\Delta r_{1}-\Delta r_{2}}\right] D \sin (\theta-\alpha)}
$$


Type: $\beta$ between the baseline direction of the two observation station and the position of the target relative to the remote station.

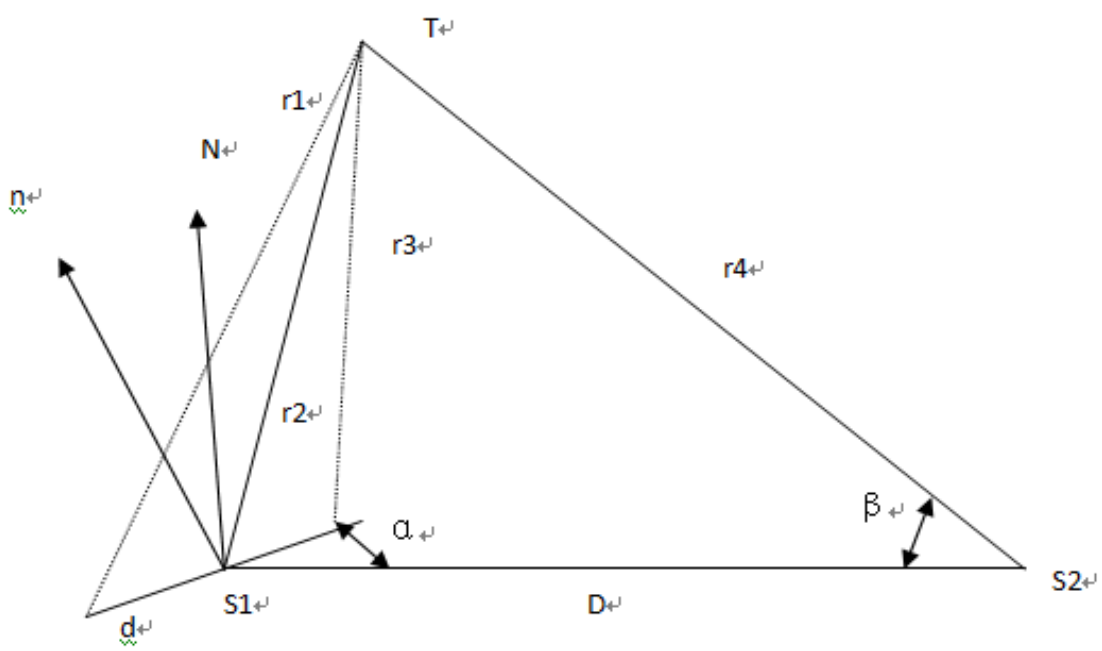

Figure 2 geometry diagram of remote observation station

The azimuth angle can be obtained by the cosine law:

$$
\beta=\cos ^{-1}\left(\frac{r_{4}^{2}+D^{2}-r_{2}^{2}}{2 r_{4} D}\right)
$$

Or by using the sine theorem:

$$
\tan \beta=\frac{r_{2} \cos (\alpha-\theta)}{D+r_{2} \sin (\alpha-\theta)}
$$

\section{Range error of remote observation station}

\section{The partial differential of the distance to the difference}

Distance measurement station ranging (15) to the difference of $\Delta \phi_{i}$ the partial differential can be rewritten as follows:

$$
\frac{\partial r_{4}}{\partial \Delta \phi_{i}}=\frac{\partial r_{4}}{\partial \Delta r_{i}} \bullet \frac{\partial \Delta r_{i}}{\partial \Delta \phi_{i}}
$$

The differential is a constant with the difference of the process:

$$
\frac{\partial \Delta r_{i}}{\partial \Delta \phi_{i}}=\frac{\lambda}{2 \pi}
$$

The partial differential of the distance ranging (15) $\Delta r_{i}$ to the differential:

$$
\frac{\partial r_{4}}{\partial \Delta r_{i}}=\frac{r_{2}}{r_{4}} \frac{\partial r_{2}}{\partial \Delta r_{i}}-\frac{D}{r_{4}}\left[\frac{\partial r_{2}}{\partial \Delta r_{i}} \sin (\theta-\alpha)+r_{2} \cos (\theta-\alpha) \frac{\partial \theta}{\partial \Delta r_{i}}\right]
$$

\section{The partial differential of the distance to the distance}

Hypothesis: 


$$
r_{2}=\frac{2 d^{2}-\Delta r_{1}^{2}-\Delta r_{2}^{2}}{2\left(\Delta r_{1}-\Delta r_{2}\right)}=\frac{p_{r}}{q_{r}}
$$

$p_{r}=2 d^{2}-\Delta r_{1}^{2}-\Delta r_{2}^{2}, q_{r}=2\left(\Delta r_{1}-\Delta r_{2}\right)$

The partial differential of the distance to the distance:

$$
\frac{\partial r_{2}}{\partial \Delta r_{i}}=\frac{1}{q_{r}^{2}}\left[q_{r} \frac{\partial p_{r}}{\partial \Delta r_{i}}-p_{r} \frac{\partial q_{r}}{\partial \Delta r_{i}}\right]
$$

$\frac{\partial p_{r}}{\partial \Delta r_{i}}=-2 \Delta r_{i}, \frac{\partial q_{r}}{\partial \Delta r_{i}}=2(-1)^{i-1}$

The partial differential of the azimuth of the near observation

Hypothesis:

$$
\sin \theta=\frac{\left(d^{2}-\Delta r_{1}^{2}\right) \Delta r_{2}+\left(d^{2}-\Delta r_{2}^{2}\right) \Delta r_{1}}{d\left(2 d^{2}-\Delta r_{1}^{2}-\Delta r_{2}^{2}\right)}=\frac{p_{\theta}}{q_{\theta}}
$$

$p_{\theta}=\left(d^{2}-\Delta r_{1}^{2}\right) \Delta r_{2}+\left(d^{2}-\Delta r_{2}^{2}\right) \Delta r_{1}$

$q_{\theta}=d\left(2 d^{2}-\Delta r_{1}^{2}-\Delta r_{2}^{2}\right)$

The differential of the deviation of the azimuth of the observation is the difference between the deviation and the deviation:

$$
\begin{gathered}
\frac{\partial \theta}{\partial \Delta r_{i}}=\frac{1}{q_{\theta}^{2} \cos \theta}\left[q_{\theta} \frac{\partial p_{\theta}}{\partial \Delta r_{i}}-p_{\theta} \frac{\partial q_{\theta}}{\partial \Delta r_{i}}\right] \\
\frac{\partial p_{\theta}}{\partial \Delta r_{i}}=d^{2}-2 \Delta r_{1} \Delta r_{2}-\Delta r_{k}^{2} \quad k=2^{2-i} \\
\frac{\partial q_{\theta}}{\partial \Delta r_{i}}=-2 d \Delta r_{i}
\end{gathered}
$$

The partial differential of the short baseline angle of the near observation station

The partial differential of the distance (15) to the angle $\alpha$ :

$$
\frac{\partial r_{4}}{\partial \alpha}=\frac{r_{2} D}{r_{4}} \cos (\theta-\alpha)
$$

The partial differential of the post station distance to the bistatic baseline

The partial differential between the two stations (15) on the baseline D:

$$
\frac{\partial r_{4}}{\partial D}=\frac{D-r_{2} \sin (\theta-\alpha)}{r_{4}}
$$

\section{Relative error of distance measurement}

According to the error estimation theory, the relative measurement error of $\mathrm{R}$ is measured. 


$$
\sigma_{r}=\frac{1}{r_{4}}\left\{\sum_{i=1}^{2}\left|\sigma_{\phi} \frac{\partial r_{4}}{\partial \Delta \phi_{i}}\right|+\left|\frac{\partial r_{4}}{\partial \alpha}\right| \sigma_{\alpha}+\left|\frac{\partial r_{4}}{\partial D}\right| \sigma_{D}\right\}
$$

Type: $\sigma_{\phi}, \sigma_{\alpha}, \sigma_{D}$ the difference between the baseline and the two stations, the root mean square error, and according to the existing engineering measurement capabilities, respectively: $\sigma_{\phi}=20^{\circ} \pi / 180, \sigma_{\alpha}=20^{\circ} \pi / 180(\mathrm{rad}), \sigma_{D}=50(\mathrm{~km})$

If you do not add a description, the distance $D=200 \mathrm{~km}$ from the remote station to the target is analyzed. To enter near probe station $r_{2}=20 \mathrm{~km}, d=1.5 \mathrm{~m}, \alpha=60^{\circ}$ 。

Through the calculation and analysis of data can be a clear explanation to increase the distance between the two stations is conducive to improve the accuracy of ranging. Simulation results show that the effect of short baseline angle of distance ranging error larger, through reduced Mae station into the target near the detection distance and to increase after the short baseline length can significantly improve accuracy of long-range laser ranging.

\section{Summary}

The mathematical analysis shows that the remote ranging accuracy is determined by the short baseline and the front and back of the front observation station, so that the short baseline length of the station can be appropriately compressed before the compression is appropriate. It can realize the miniaturization of the front range observation station.

\section{References:}

[1] JIAO Y S, DU J, WANG X M, et al. H1 state feedback control for UAV Maneuver trajectory tracking[C] //Proceedings of 2010 International Conference on Intelligent Control and Information Processing.Dalian, China: IEEE, 2010, 8: 253 - 257.

[2] AHMED B, POTA H R, MATT G. Flight control of a rotary wing UAV using backstepping[J]. International Journal of Robust and Nonlinear Control, 2010, 20(6): 639 - 658.

[3] LEE C T, TSAI C C. Adaptive backstepping integral control of a small-scale helicopter for airdrop missions[J]. Asian Journal of Control,2010, 12(4): 531 - 541.

[4] SANCHEZ E N, BECERRA H M, VELEZ C M. Combining fuzzyand PID control for an unmanned helicopter[C] //2005 Annual Meetingof the North American Fuzzy Information Processing Society. Detroit,USA: IEEE, 2005, 6: 235 - 240.

[5] ISIDORI A, MARCONI L, SERRANI A. Robust nonlinear motion control of a helicopter[J]. IEEE Transactions on Automatic Control,2003, 48(3): 413 - 426.

[6] MAHONY R, HAMEL T. Robust trajectory tracking for a scale model autonomous helicopter[J]. International Journal of Robust and Nonlinear Control, 2004, 14(12): 1035 - 1059.

[7] SHIN J, NONAMI K, FUJIWARA D, et al. Model-based optimal attitude and position control of a small-scale unmanned helicopter[J].Robotica, 2005, 23(1): 51 - 63. 